\title{
Disrupting hedgehog and WNT signaling interactions promotes cleft lip pathogenesis
}

\author{
Hiroshi Kurosaka, ${ }^{1}$ Angelo lulianella, ${ }^{2}$ Trevor Williams, ${ }^{3}$ and Paul A. Trainor ${ }^{1,4}$
}

\begin{abstract}
${ }^{1}$ Stowers Institute for Medical Research, Kansas City, Missouri, USA. ${ }^{2}$ Department of Medical Neuroscience, Faculty of Medicine, Dalhousie University, Halifax, Nova Scotia, Canada. ${ }^{3}$ Department of Craniofacial Biology and Cell and Developmental Biology, University of Colorado Anschutz Medical Campus, Aurora, Colorado, USA. ${ }^{4}$ Department of Anatomy and Cell Biology, University of Kansas Medical Center, Kansas City, Kansas, USA.
\end{abstract}

\begin{abstract}
Cleft lip, which results from impaired facial process growth and fusion, is one of the most common craniofacial birth defects. Many genes are known to be involved in the etiology of this disorder; however, our understanding of cleft lip pathogenesis remains incomplete. In the present study, we uncovered a role for sonic hedgehog $(\mathrm{SHH})$ signaling during lip fusion. Mice carrying compound mutations in hedgehog acyltransferase (Hhat) and patched1 (Ptch1) exhibited perturbations in the SHH gradient during frontonasal development, which led to hypoplastic nasal process outgrowth, epithelial seam persistence, and cleft lip. Further investigation revealed that enhanced SHH signaling restricts canonical WNT signaling in the lambdoidal region by promoting expression of genes encoding WNT inhibitors. Moreover, reduction of canonical WNT signaling perturbed $\mathrm{p} 63$ /interferon regulatory factor 6 (p63/IRF6) signaling, resulting in increased proliferation and decreased cell death, which was followed by persistence of the epithelial seam and cleft lip. Consistent with our results, mutations in genes that disrupt SHH and WNT signaling have been identified in both mice and humans with cleft lip. Collectively, our data illustrate that altered SHH signaling contributes to the etiology and pathogenesis of cleft lip through antagonistic interactions with other gene regulatory networks, including the canonical WNT and P63/IRF6 signaling pathways.
\end{abstract}

\section{Introduction}

Craniofacial development depends on the proper growth and fusion of distinct facial processes during embryogenesis. Perturbation of either developmental event can lead to facial cleft phenotypes $(1,2)$. It is well known that, during facial process fusion, epithelial cells at the tip of each process are eliminated by several mechanisms, including cell death both in lip and secondary palate fusion $(2,3)$ and epithelial mesenchymal transition during secondary palate fusion $(4,5)$. One of the most common sites of fusion failure is at the lambdoidal junction between the maxillary process and medial nasal process (MNP) and lateral nasal process (LNP). Failure or delay of lambdoidal junction development or fusion leads to cleft lip with or without cleft palate (CL/P). This is one of the most common birth defects, with an incidence of 1 in 700 newborns (6). Many signaling pathways are known to be associated with the etiology of CL/P. Sonic hedgehog (SHH) signaling, for example, is indispensable for proper craniofacial development, and perturbation of this pathway is associated with $\mathrm{CL} / \mathrm{P}$ in certain human congenital diseases, such as nevoid basal cell carcinoma syndrome and holoprosencephaly $(7,8)$. Furthermore conditional epithelial or mesenchymal elimination of SHH signaling results in palatal fusion defects in mice $(9,10)$, as does chemical inhibition of SHH signaling (11). These results strongly suggest that $\mathrm{SHH}$ signaling plays an important role in CL/P etiology; however, the detailed molecular mechanism remains to be elucidated.

Interestingly, disruption of canonical WNT signaling is also associated with CL/P both in mice and humans (12-14). WNT signaling functions as a positive regulator of $\mathrm{p} 63$ and interferon regulatory factor 6 (IRF6) (15), which regulates proper facial process fusion by controlling epithelial cell proliferation and differ-

Conflict of interest: The authors have declared that no conflict of interest exists. Citation for this article: J Clin Invest. 2014;124(4):1660-1671. doi:10.1172/JCI72688. entiation $(16,17)$. In the present study, we discovered that mouse embryos, which carry compound mutations in hedgehog acyltransferase (Hhat) (Hhatcreface) (18) and patched1 (Ptch1 wiggable) (19), exhibit cleft lip in association with expanded SHH signaling during craniofacial development. We also discovered that expanded SHH signaling concomitantly inhibited canonical WNT signaling through the regulation of WNT inhibitory genes. Furthermore, we uncovered compromised p63/IRF6 activity, altered domains of cell death, and increased cell proliferation. Collectively, this led to persistence of the epithelial seam in the lambdoidal region and pathogenesis of cleft lip. Therefore, our results have uncovered important signaling interactions between the HH and WNT pathways during craniofacial development, which underpin the etiology and pathogenesis of cleft lip.

\section{Results}

Hhatcreface Ptch1 wiggable embryos exhibit multiple developmental defects, including cleft lip. Hhat encodes an acyltransferase that is responsible for modifying hedgehog $(\mathrm{HH})$ proteins through the addition of palmitic acid. Disruption of Hhat diminishes palmitoylation of SHH, which perturbs its secretion and long-range activity $(20,21)$. Consistent with these findings, Hhat ${ }^{\text {creface }}$ mice exhibit severe craniofacial defects, which are caused by a disruption of $\mathrm{HH}$ signaling (18). A holoprosencephaly phenotype is clearly evident in association with small head size in E13.5 Hhatcreface embryos (Figure 1C) compared with control embryos (Figure 1A). Furthermore, ventral views of the craniofacial skeleton reveal severe bone and cartilage defects in E16.5 Hhatcreface embryos (Figure 1F) compared with controls (Figure 1D). In particular, the palatal process of premaxilla bone is missing and both sides of the maxilla bone are fused, resulting in a single bone fragment at the center of the cranial base in Hhat creface embryos (Figure 1F). These phenotypes are consistent with a loss of SHH signaling (18). 


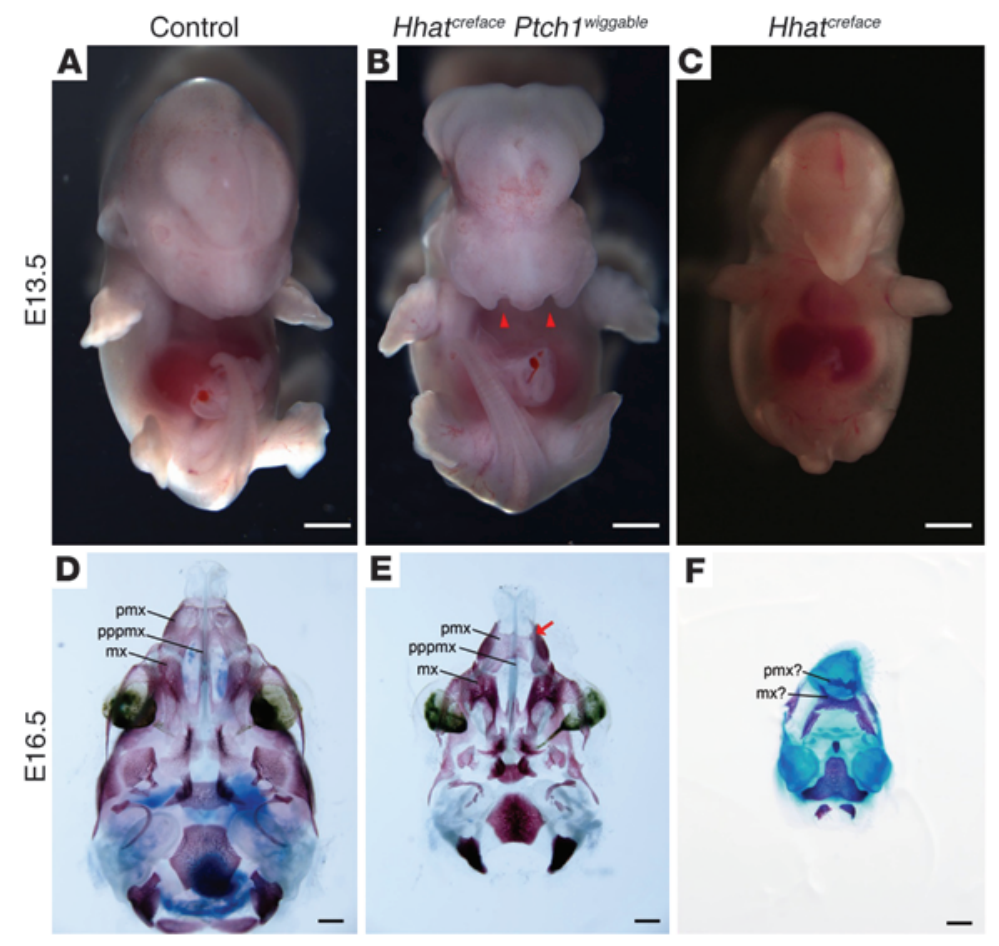

\section{Figure 1}

Hhatcreface Ptch1 ${ }^{\text {wiggable }}$ embryos exhibit cleft lip. (A-C) Whole body image of E13.5 control, Hhatcreface Ptch1wiggable, and Hhatcreface embryos. (B) Obvious cleft lip phenotype could be observed in Hhatcreface Ptch1wiggable embryos (red arrowheads). (C) Hhatcreface embryos showed smaller head size than (A) control embryos. (D-F) Whole head Alcian blueAlizarin red skeletal staining of embryos of the indicated genotypes at E16.5. (E) Hhatcreface Ptch1wiggable embryos showed bone cleft in premaxilla (red arrow). (F) Hhatcreface embryos showed severely affected skeletal structures compared with (D) control embryos. pmx, premaxilla; pppmx, palatal process of premaxilla; $\mathrm{mx}$, maxilla. Scale bars: $1 \mathrm{~mm}$.
In contrast, $\mathrm{PTCH} 1$ is a receptor for $\mathrm{HH}$ ligands and works as a repressor of $\mathrm{SHH}$ signaling in the absence of $\mathrm{SHH}$ ligand. Consistent with this role, disruption of Ptch1 in mice results in an elevation of SHH signaling (22). Through an $\mathrm{N}$-ethyl- $\mathrm{N}$-nitrosourea mutagenesis screen in mice (19), we generated a novel allele of Ptch1. Genome sequencing revealed a T to A nucleotide change in intron 15 of Ptch1, which created a new splice acceptor site, resulting in a premature stop codon in exon 16 and generation of a truncated protein. Ptch1 $1^{\text {wiggable }}$ embryos die in utero at around E12.0 as a result of various defects, including open neural tube and hypertelorism of the face (19). These phenotypes are consistent with a gain of function in $\mathrm{HH}$ signaling. In an effort to mutually rescue the Hhat treface and Ptch1 $1^{\text {wiggable }}$ phenotypes, we crossed Hhat reface mice with Ptch1 ${ }^{\text {wiggable }}$ mice to generate double homozygotes (Hhat ${ }^{\text {creface }}$ Ptch $1^{\text {wiggable }}$ mice) and partially restored head morphology and craniofacial structures. Interestingly, however, these embryos presented with cleft lip (Figure 1B) and fissure of the premaxilla bone at E16.5 (Figure 1E), implying that HHAT and PTCH1 played an important role in regulating $\mathrm{HH}$ signaling during lip development.

MNPs and LNPs fail to fuse in Hhat ${ }^{\text {creface }}$ Ptch $1^{\text {wiggable }}$ embryos. To investigate the mechanism underlying the pathogenesis of cleft lip in Hhat ${ }^{\text {creface }}$ Ptch1 $1^{\text {wiggable }}$ mice, we explored the onset of developmental anomalies in individual and compound mutants (Figure 2). At E10.0, Ptch1 wiggable embryos showed severe craniofacial defects, including open neural tube, together with frontonasal and bran-

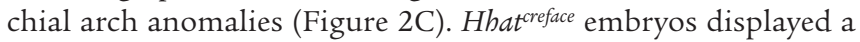
hypoplastic first branchial arch and minor frontonasal process (FNP) deformities (Figure 2D). Hhat ${ }^{\text {creface }}$ Ptch1 wiggable embryos similarly presented with a smaller FNP (Figure 2B) compared with that of control embryos (Figure 2A). By E11.0, wild-type embryos exhibited prominent MNPs and LNPs (Figure 2E). In contrast, Ptch1 wiggable embryos displayed enlarged maxillary processes, but neither the MNPs nor LNPs could be readily distinguished at this stage (Fig- ure 2G). Hhat treface embryos showed facial deformities represented by reduced spacing between the bilateral nasal slits with hypoplastic maxillary and mandibular processes (Figure $2 \mathrm{H}$ ). Hhat ${ }^{\text {creface }}$ Ptch1 ${ }^{\text {wiggable }}$ embryos showed a substantial restoration of facial development compared with each single mutant; however, these double mutants still displayed deficient MNP and LNP growth (Figure 2F). By E11.5, the MNP and LNP fused at the lambdoidal region in control embryos to form the future lip and primary palate (Figure 2, I, M, and Q). In contrast, Ptch1 ${ }^{\text {wiggable }}$ embryos displayed severe defects in nasal process growth as well as nasal epithelium invagination (Figure 2, $\mathrm{K}$ and $\mathrm{O}$, and Supplemental Figure 1, A-C; supplemental material available online with this article; doi:10.1172/ JCI72688DS1). Substantial MNP defects could also be observed in Hhat ${ }^{\text {creface }}$ embryos in the form of a single nasal slit at the midline of the face (Figure 2, L and P, and Supplemental Figure 1, D-F). E11.5 Hhat ${ }^{\text {creface }}$ Ptch 1 wiggable embryos showed considerable outgrowth of the MNPs and LNPs compared with that at earlier stages; however, the failure of these processes to fuse left a large gap that leads to cleft lip and primary cleft palate (Figure 2, J, N, and R).

Altered balance of cell proliferation and cell death in the nasal processes of

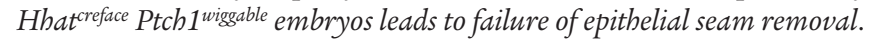
Epithelial cell removal via cell death is a crucial event during facial process fusion (2). In the case of normal development, epithelial seam cells between the FNP and MNP break down via loss of e-cadherin (ECAD) expression and apoptosis, as shown by TUNEL staining, which resulted in formation of a mesenchymal bridge (Figure 3, A-C, white arrow). Epithelial seam cell death usually occurs just at the tip of the fusing processes, which is similar to the process underlying secondary palate fusion. Hhat ${ }^{\text {creface }} P t c h 1^{\text {wiggable }}$ embryos exhibited reduced apoptosis in the seam where epithelial cell break down should normally occur (Figure 3F, white arrow). Together with the persistence of ECAD-positive cells, this lead to the failure to form a mesenchymal bridge (Figure 3, D-F, white 

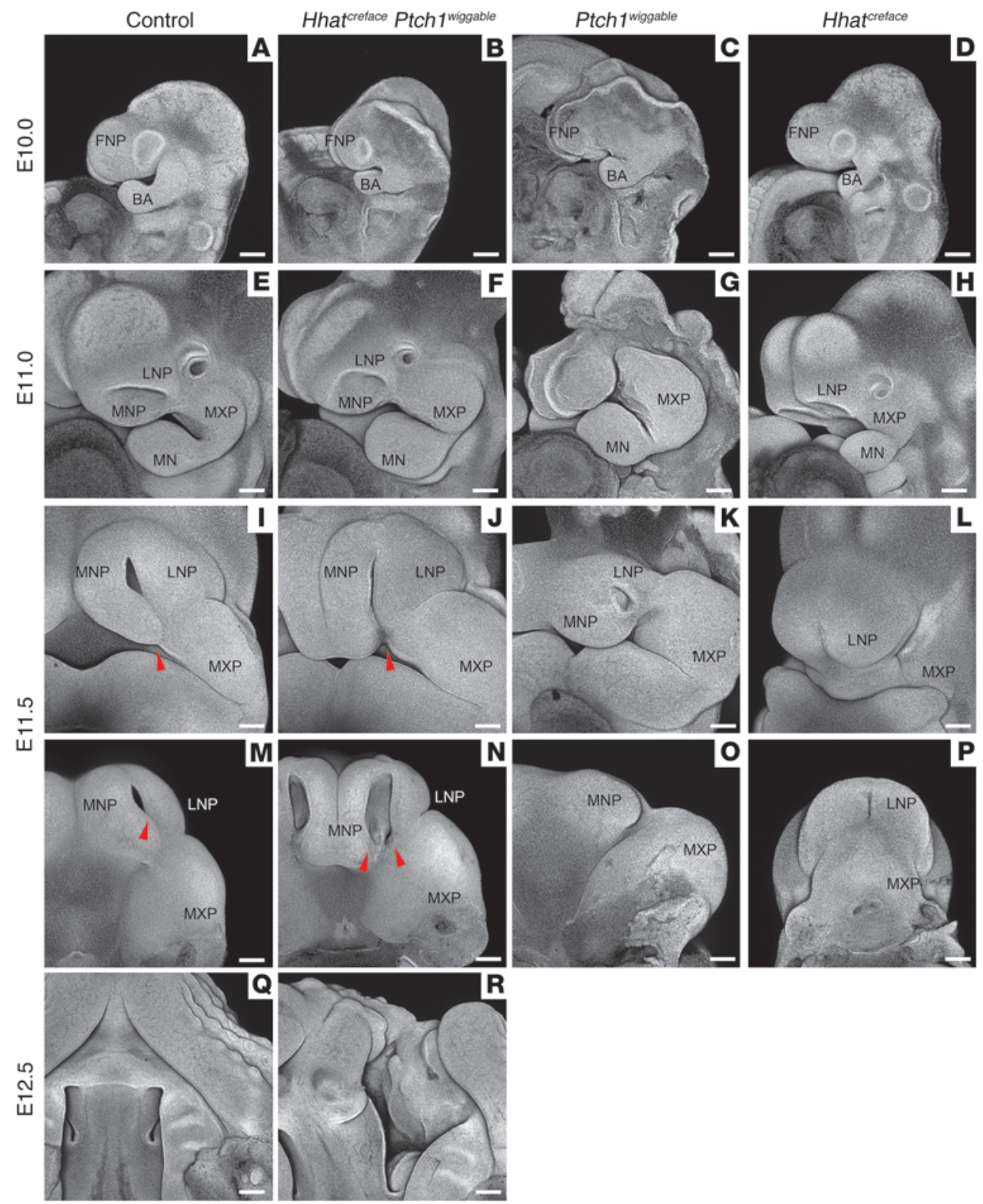

\section{Figure 2}

Mutation in Hhat and Ptch1 genes modifies facial process growth and fusion, as shown by nuclear fluorescent imaging. (A-H) Lateral view of embryos, with genotypes shown at the top and embryonic stages shown at the left. (A-D) At E10.0, each embryo developed morphologically similar FNPs. (E) At E11.0, MNP and LNP begin to develop in control embryos. (F and G) Hhatcreface Ptch1wiggable and Ptch1wiggable embryos developed hypoplastic MNPs and LNPs. (H) Hhatcreface embryos showed shorter distance between left and right nasal pit. (I-L) Frontal view of embryos of each indicated genotype at E11.5. (I and J) Obvious fissure exists between MNP and LNP in Hhatcreface Ptch1wiggable embryos compared with that in control embryos (red arrowheads). (K) Ptch1wiggable embryos showed severely affected FNPs. (L) MNP was not detectable from an external view of Hhatcreface embryo head. (M-R) Ventral view of maxillary complex. ( $\mathbf{M}$ and $\mathbf{N}$ ) MNP and LNP failed to fuse in Hhatcreface Ptch1wiggable embryos and left larger gap between those processes compared with control embryos (red arrowheads). ( $\mathbf{Q}$ and R) At E12.5, Hhatcreface Ptch1 wiggable embryos showed obvious cleft lip and primary palate compared with control embryos. MXP, maxillary process; BA, branchial arch; MN, mandible. Scale bars: $200 \mu \mathrm{m}$. arrow). Conversely, we found very few mitotic cells in the epithelial seam in control embryos, as measured by phospho-histone $\mathrm{H} 3$ immunostaining (Figure 3, G-I and O). However, there was a considerable increase in phospho-histone H3-labeled cells in Hhat ${ }^{\text {creface }}$ Ptch1 ${ }^{\text {wiggable e }}$ embryos (Figure 3, J-L, yellow arrowheads, and $\mathrm{O}$ ). These results suggest that during normal development epithelial seam cells stop proliferating and undergo apoptosis in order to break down epithelial cells as part of the mechanism of lip fusion. In contrast, the ECAD-positive epithelial cell layer (Figure 3, J-L) was thicker in Hhatcreface Ptch1 ${ }^{\text {wiggable }}$ embryos than in control embryos (Figure 3, G-I). We also assessed the persistence of periderm cells using an antibody that recognizes SSEA1 protein. Interestingly, the intensity of SSEA1 was stronger in the nasal process epithelia of Hhat ${ }^{\text {creface }}$ Ptch $1^{\text {wiggable }}$ embryos (Supplemental Figure 2, B and D) compared with that of control embryos (Supplemental Figure 2, A and C). These results suggest a persis- tence of periderm cells in the nasal processes of Hhatcreface Ptch1 wiggable embryos in association with the failure of lip fusion.

The gradient of SHH signaling during facial morphogenesis was perturbed in each mutant. Since HHAT and PTCH1 are the key regulators of SHH signaling $(18,21,22)$, we hypothesized that each mutant should have altered SHH signaling during facial morphogenesis. Hence, we assessed $S h b$ mRNA expression via in situ hybridization in developing E9.5 embryos. Regardless of the genotype, each embryo showed strong Shb mRNA expression in the notochord and floor plate (Figure 4, A-D). We also observed that there was no Shb mRNA activity in the MNPs and LNPs among all the embryos at E11.0 (Figure 4, E-H). We next performed Ptch1 and Gli1 in situ hybridization to document the degree of spatial perturbation of SHH signaling, since the activity of these genes is widely considered as representative of the gradient and range of SHH signaling. In control embryos, Ptch1 as well as Gli1 mRNA were expressed inten- 

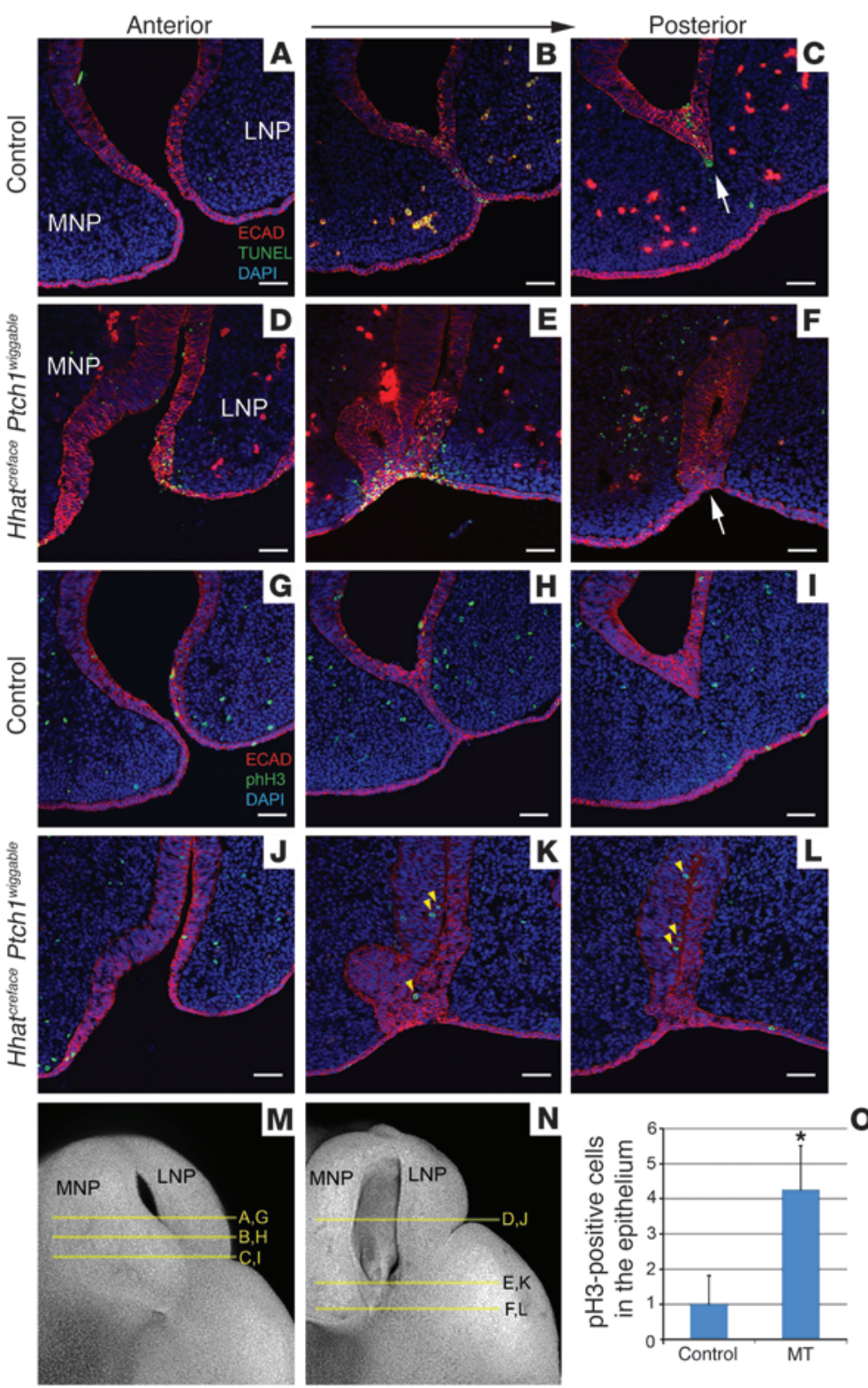

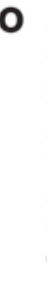

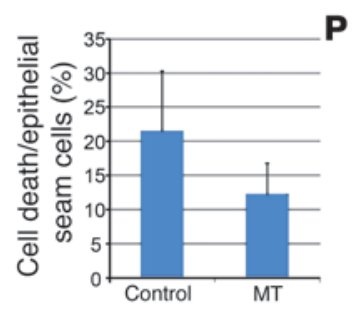

\section{Figure 3}

Cell death and proliferation in epithelial seams of E11.5 Hhatcreface Ptch 1 wiggable embryos. (A-F) TUNEL staining (green) with ECAD immunolabeling (red) in E11.5 control and Hhatcreface Ptch1 wiggable embryo FNPs. (A-C) Control embryo epithelial seam cells underwent cell death and diminished ECAD immunostaining to remove the epithelial cells (white arrow). (D-F) Hhatcreface Ptch1wiggable embryos showed thicker epithelial seam and lacked proper cell death (white arrow). (G-L) Immunolabeling of phospho-histone $\mathrm{H} 3$ (green) and ECAD (red) in E11.5 control and Hhatcreface Ptch1wiggable embryo FNPs. (G-I) Very few proliferating cells could be detected in control embryo epithelial seams. (J-L) In contrast, increased cell proliferation was detected in Hhatcreface Ptch1wiggable embryo epithelial seam cells (yellow arrowheads). (0) Statistical analysis showed significant difference in number of proliferating cells between Hhatcreface Ptch1wiggable (MT) and control embryos. (P) Percentage of apoptotic cells in epithelial seam cells of control and Hhatcreface Ptch1wiggable embryos. ${ }^{*} P<0.05$, Student's $t$ test. Data are represented as mean \pm SEM. ( $\mathbf{M}$ and $\mathbf{N}$ ) Each yellow line indicates which plane was used in these images. Scale bars: $50 \mu \mathrm{m}$. sively in the mesenchyme and epithelia of the MNPs, with gradually reduced signal and an absence of expression in the LNPs (Figure 4, I and M). As expected, Ptch $1^{\text {wiggable }}$ embryos showed significantly stronger and expanded Ptch1 and Gli1 expression throughout the whole FNP, as evidenced by both in situ hybridization and RNA sequencing (RNA-seq) (Figure 4, K and O, and Supplemental Figure 3). In contrast, Hhat treface embryos showed considerably reduced expression of Ptch1 and Gli1 (Figure 4, L and P, and Supplemental Figure 3, B and C), which is consistent with a diminished range and gradient of SHH signaling (18). Craniofacial structures are partially restored in Hhat ${ }^{\text {creface }}$ Ptch1 wiggable double-homozygous embryos, and, consistent with this, the quantity of Ptch1 and Gli1 mRNA in the maxillary complex was returned to normal, as assessed by RNA-seq (Supplemental Figure 3, B and C). However, most importantly, the distribution of gene activity in Hhat ${ }^{\text {reface }}$ Ptch1 wiggable embryos was not fully restored to a wild-type pattern, as ectopic expression remained in the LNP (Figure 4, J and N).
Complementary SHH and canonical WNT signaling during craniofacial development. To investigate the gene regulatory network governing the cleft lip phenotype observed in double-mutant embryos, we performed RNA-seq analyses and identified a downregulation of canonical WNT signaling (Supplemental Figure 3, G-I) in association with enhanced SHH signaling (Supplemental Figure 3, B and C). We also discovered that cells that respond to canonical WNT signaling localize in complementary domains to SHH-responding cells in developing FNPs, as observed through $L a c Z$ reporter expression in TOPgal (23) and Ptch1-LacZ (22) embryos, respectively (Supplemental Figure 4, A-F). These results suggest that SHH signaling and canonical WNT signaling regulate complementary territories during proper craniofacial morphogenesis and patterning. Canonical WNT signaling is inbibited by SHH during frontonasal development. To further investigate the relationship between $\mathrm{SHH}$ and canonical WNT activity during craniofacial morphogenesis, we crossed each mutant to TOPgal mice and used LacZ expression as a 

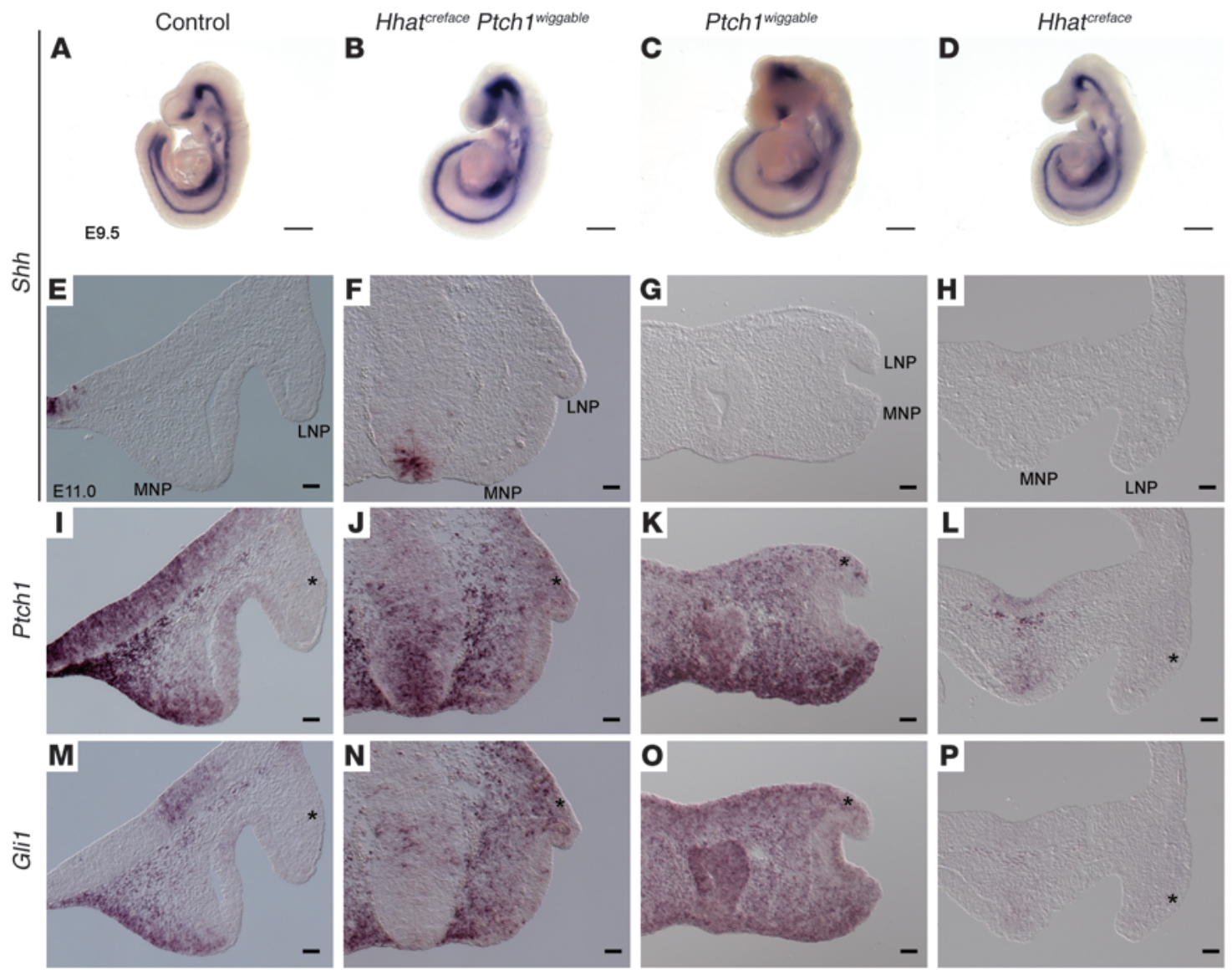

\section{Figure 4}

SHH signaling is altered by mutation of Hhat and Ptch1 genes. (A-D) Lateral view of WISH of Shh in E9.5 embryos. All embryos showed continuous Shh mRNA expression along the notochord and floor plate. (E-P) In situ hybridization of Shh, Ptch1, and Gli1 using E11.0 frontal sections of embryo heads of each indicated genotype. (E-H) None of them showed Shh transcript in MNPs or LNPs. (I and M) In control embryos, Ptch1 and Gli1 transcripts showed highest expression in the middle of the face and gradually reduced expression toward the outside and were absent from the LNP (asterisks). (L and $\mathbf{P})$ Hhatcreface embryos showed considerable reduction of both genes at the center of developing face. (J, K, N, and O) Hhatcreface Ptch1wiggable and Ptch1wiggable embryos both showed expanded expression of Ptch1 and Gli1 and expression of these genes could observed in the LNP (asterisks). Scale bars: $500 \mu \mathrm{m}$ (A-D); $50 \mu \mathrm{m}$ (E-P).

read out of canonical WNT signaling. At E10.0, Hhatreface embryos expressed LacZ in the LNPs (Figure 5D, red arrowhead), while embryos of the other genotypes did not (Figure 5, A-C). At E10.5, a considerable reduction in $L a c Z$ expression was detected in Ptch1 $1^{\text {wiggable }}$ embryos (Figure 5G) as well as in Hhat ${ }^{\text {creface }}$ Ptch1 $1^{\text {wiggable }}$ embryos (Figure 5F) compared with that in control embryos (Figure 5E). In contrast, Hhat ${ }^{\text {creface }}$ embryos showed expanded WNT activity in the LNPs (Figure 5H). By E11.0, the stage when the LNP and MNP fuse, strong $\mathrm{LacZ}$ activity could be observed at the lambdoidal junction in control embryos (Figure 5I, red arrowhead), while Hhat ${ }^{\text {creface }}$

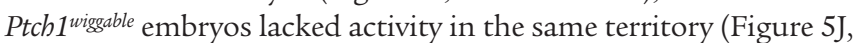
red arrowhead). Furthermore, histological sections clearly indicated that LacZ expression in Hhat ${ }^{\text {creface }}$ Ptch 1 wiggable embryos (Figure $5 \mathrm{~N}$, red arrowhead) was considerably reduced, especially in the olfactory epithelia, compared with that in control embryos (Figure 5M, red arrowhead). These finding are consistent with the results from our RNA-seq analysis (Supplemental Figure 3, D-I) confirming the spatial reduction in canonical WNT signaling. We also performed whole embryo culture using TOPgal embryos in the presence of a
SHH signaling activator (purmorphamine). As expected, embryos cultured with purmorphamine showed a considerable reduction in WNT activity (LacZ) in the lambdoidal region (Supplemental Figure 5B) compared with that in DMSO-treated embryos (Supplemental Figure $5 \mathrm{~A}$ ). These results clearly indicate that $\mathrm{SHH}$ signaling restricts canonical WNT signaling during frontonasal development.

Canonical WNT inbibitor activity is modulated by SHH signaling. Since canonical WNT signaling is restricted by SHH signaling, we hypothesized that this may occur via activation of WNT inhibitors. From our RNA-seq data, we speculated that Vax1 and Sfrps might be modified by altered SHH signaling. To test our hypothesis, we performed in situ hybridization for those genes in our mutants (Figure 6). Interestingly, the Vax1 expression domain was shifted distally in the nasal epithelia of Hhat ${ }^{\text {creface }}$ Ptch1 wiggable embryos (Figure 6B) compared with that of control embryos (Figure 6A). Similar to Vax1, the expression pattern of Sfrp1 was also changed in compound mutant embryos (Figure 6, E and F). Furthermore, Sfrp2 and Frzb (also known as Sfrp3), which are primarily expressed in mesenchyme cells in control embryos (Figure 6, I and M, aster- 

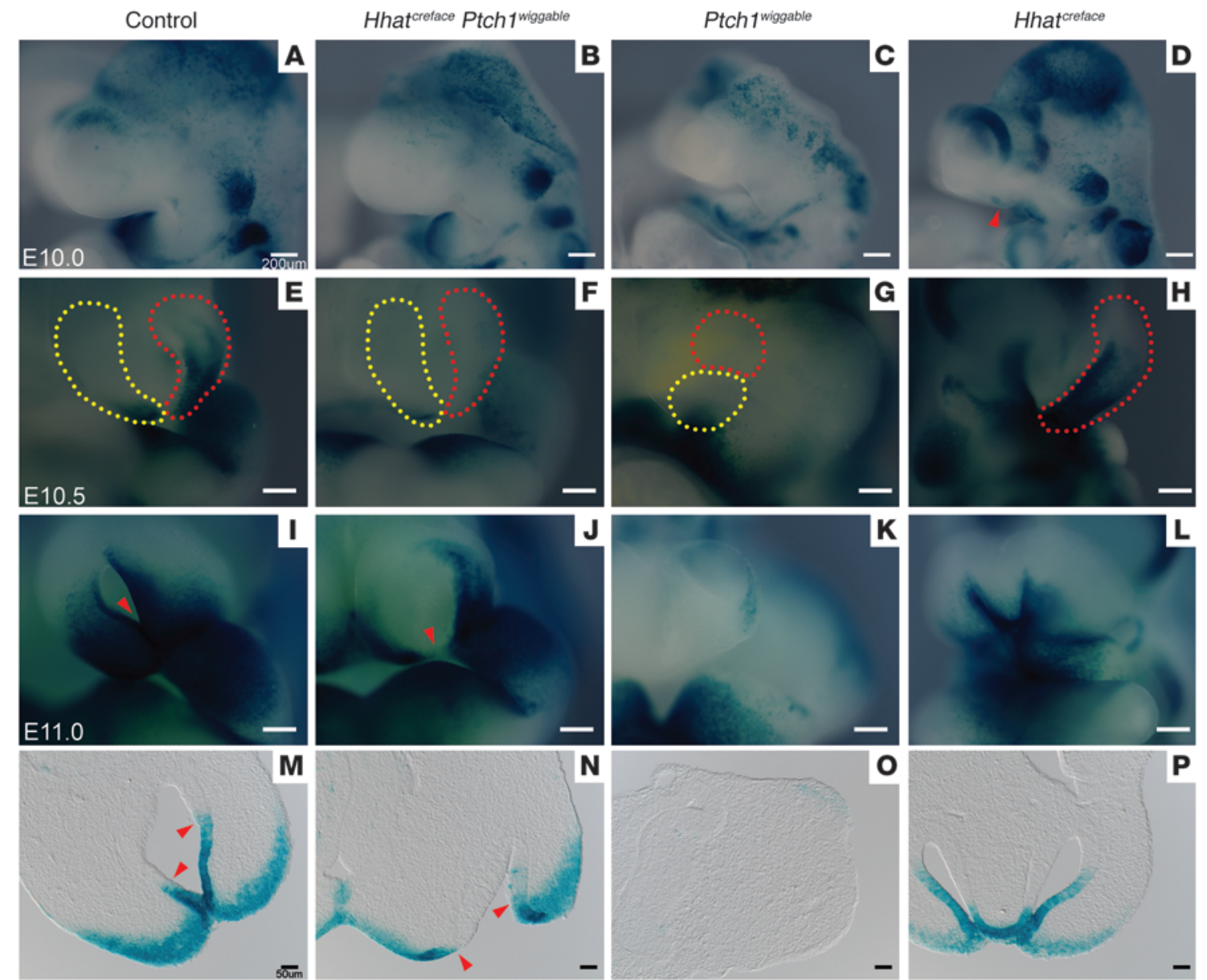

\section{Figure 5}

Canonical WNT signaling is affected by altering SHH signaling during craniofacial development. (A-D) Lateral views of heads of LacZ-stained E10.0 embryos of the indicated genotypes crossed with TOPgal mice (orientation is the same as that shown in Figure 2, A-D). (D) Hhatcreface embryos showed LacZ expression at LNP (red arrowhead), (A-C) while none of other embryos showed it. (E-H) Ventral views of E10.5 embryo MNPs (yellow dotted lines) and LNPs (red dotted lines) of each indicated genotype (orientation is the same as that shown in Figure 2, I-L). (F and G) Hhatcreface Ptch1wiggable and Ptch1wiggable embryos both showed noticeable reduction of LacZ expression in MNPs and LNPs compared with controls (E). (H) Hhatcreface embryos showed expanded LacZ expression in LNP. (I-L) Ventral views of E11.0 FNPs of each indicated genotype (orientation is the same as that shown in Figure 2, I-L). (I) Control embryos strongly expressed LacZ at the lambdoidal region where the MNP and LNP fuse (red arrowhead). (J) Hhatcreface Ptch1 wiggable embryos showed reduced expression of LacZ at the lambdoidal region (red arrowhead). (K) Ptch1 1wiggable embryos showed even more reduction at FNPs. (M-P) Frontal sections of LacZ-stained embryos at E11.0. (M) Control embryos showed strong LacZ expression at epithelial seam and nasal epithelium (red arrowheads). (N) Hhatcreface Ptch 1 wiggable embryo LacZ expression was restricted compared with that of control embryos (red arrowheads). (0) Ptch1wiggable embryos showed very weak LacZ expression, and (P) Hhatcreface mice still maintained LacZ expression in FNPs. Scale bars: $200 \mu \mathrm{m}(\mathbf{A}-\mathbf{L}) ; 50 \mu \mathrm{m}(\mathbf{M}-\mathbf{P})$.

isks), exhibited elevated expression in Hhat ${ }^{\text {creface }}$ Ptch $1{ }^{\text {wiggable }}$ embryos (Figure 6, J and $\mathrm{N}$, asterisks). These results suggest that during craniofacial morphogenesis $\mathrm{SHH}$ signaling and its intersection with WNT signaling can be modulated by the distinct expression of individual WNT inhibitors in the facial processes.

Reduced canonical WNT signaling affects p63/IRF6 activity at the lambdoidal junction. TP63 is a member of the TP63 family of genes, and mutations in TP63 are known to be responsible for causing CL/P in mice and humans with certain syndromic diseases $(24,25)$. Recently, several studies have shown that the p63/IRF6 signaling pathway plays critical roles in palatal growth and fusion $(16,17)$. Furthermore, canonical WNT signaling functions upstream of this signaling cascade (15). We therefore hypothesized that SHH signaling-associated cleft lip occurred via disruption of WNT-medi- ated p63/IRF6 signaling. Via in situ hybridization, we found p63/ IRF6 signaling noticeably inhibited at the lambdoidal junction in Ptch1 wiggable embryos (Figure 7, C and G, and Supplemental Figure 6,

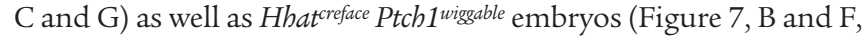
and Supplemental Figure 6, B and F) compared with that in control embryos (Figure 7, A and E, and Supplemental Figure 6, A and E). In contrast Hhat ${ }^{\text {creface }}$ embryos exhibited expanded expression (Figure 7, D and H, and Supplemental Figure 6, D and H). These results are consistent with the results from our TOPgal activity studies in each mutant and suggest that modulating $\mathrm{SHH}$ signaling alters canonical WNT signaling, which in turn affects the p63/IRF6 signaling pathway in the pathogenesis of CL/P (Figure 8).

Tfap2 a expression is diminished in the FNPs in response to altered $\mathrm{SHH}$ signaling. During craniofacial development, cranial neural crest 

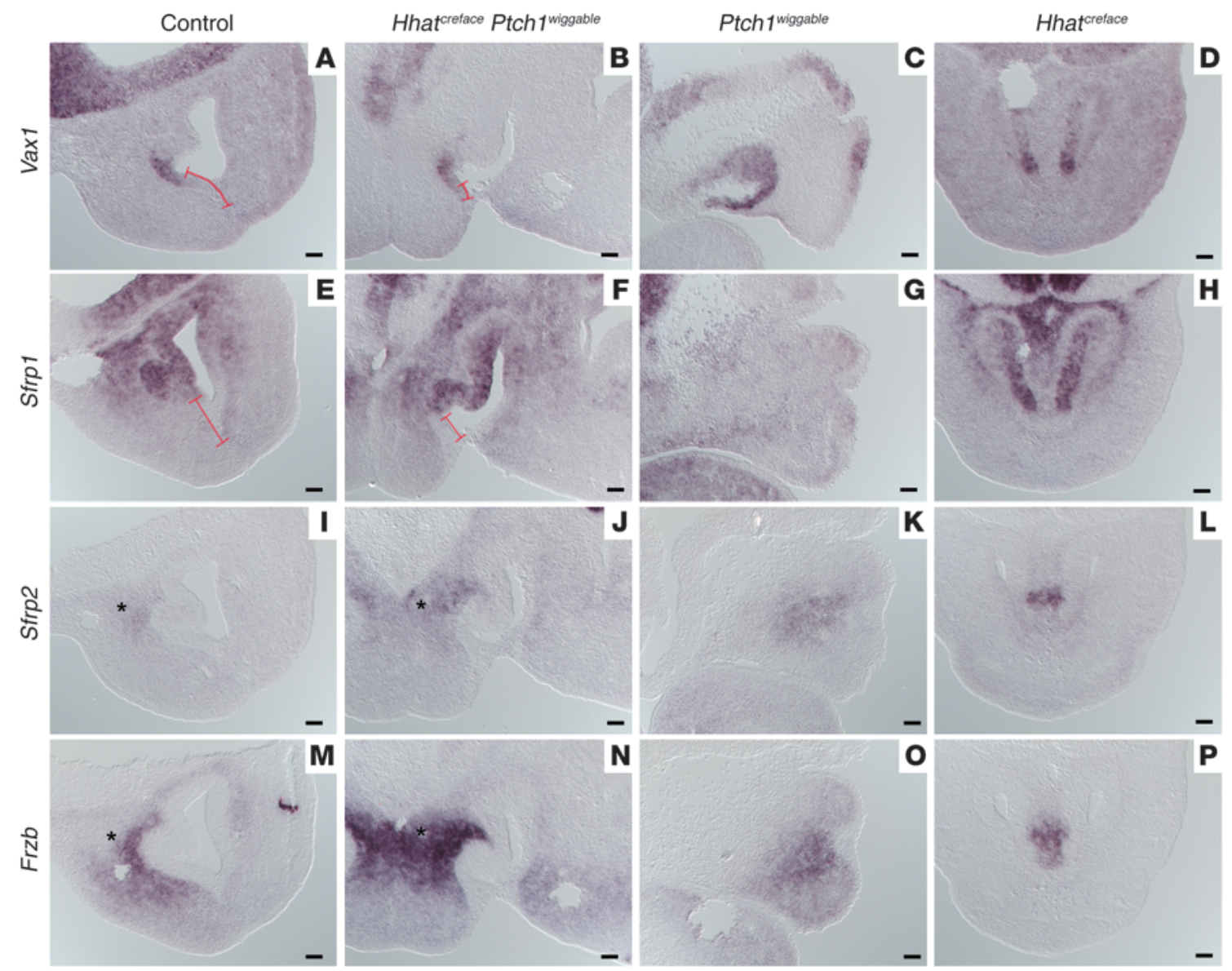

\section{Figure 6}

Canonical WNT inhibitory gene expression is modulated by SHH signaling. Expression of WNT inhibitory genes (A-D) Vax1, (E-H) Sfrp1, (I-L) Sfrp2, and (M-P) Frzb in E11.5 head frontal sections of each indicated genotype. (B) The expression domain of Vax1 shifted distally in Hhatcreface Ptch1wiggable embryos compared with that in control embryos (A; red lines). Similar to Vax1, (F) Sfrp1 changed its expression distally in Hhatcreface Ptch1wiggable embryos (E) compared with that in control embryos (red lines). (I and M) Sfrp2 and Frzb expression in mesenchyme in control embryos (asterisks) and ( $\mathbf{J}$ and $\mathbf{N}$ ) enhanced expression in Hhatcreface Ptch1 wiggable embryos (asterisks). Scale bars: $50 \mu \mathrm{m}$.

cells (CNCs) play critical roles in producing cartilage, bone, and connective tissues as well as regulating facial patterning (26-28). SHH signaling has been shown to be a critical regulator of CNC development (29). Hence, we assessed the formation and patterning of CNCs via in situ hybridization using Crabp1, Snail1, and Tfap $2 a$ as general markers of CNCs. Both Crabp1 and Snail1 showed equivalent expression patterns in the FNPs among all genotypes (Supplemental Figure 7). However, the activity of Tfap2a, which is a transcription factor that directly binds to the Irf6 promoter (30), was diminished in the FNPs in Ptch1 wiggable embryos (Figure 7K and Supplemental Figure 6K). Additionally, Tfap2a expression was noticeably reduced in Hhatereface Ptch1 wiggable embryos (Figure 7J and Supplemental Figure 6J) compared with that in controls (Figure 7I and Supplemental Figure 6I). We confirmed that TFAP2A protein is expressed in the epithelial seam in association with diminished ECAD in E11.5 control embryos (Supplemental Figure 6M). Interestingly, TFAP2A activity in the epithelial seams of Ptch1 wiggable and Hhat ${ }^{\text {refface }}$ Ptch $1^{\text {wiggable }}$ mouse embryos was considerably reduced, correlating with persistent ECAD activity (Supplemental Figure 6, $\mathrm{N}$ and $\mathrm{O}$ ). These results strongly suggest that altered or expanded SHH signaling during craniofacial development can affect the expression of Tfap2a in CNCs and TFAP2A in epithelial seam cells, which directly modifies IRF6 activity and contributes to epithelial seam persistence in the pathogenesis of cleft lip (Figure 8).

\section{Discussion}

Disruption of the SHH signaling gradient during craniofacial development results in $C L / P$ via persistence of epithelial cells between facial processes. Disruption of SHH signaling is associated with craniofacial defects, such as holoprosencephaly (loss of function) and nevoid basal cell carcinoma syndrome (gain of function) (31). The fact that both of these disorders present with $\mathrm{CL} / \mathrm{P}$ highlights the indispensable role of SHH signaling during FNP growth and fusion $(8,32)$. We recently reported 2 novel mouse lines, both of which exhibit craniofacial defects caused by either reduced (Hhatcreface) (18) or enhanced (Ptch1wiggable) (19) HH signaling, respectively. Interestingly, double-homozygous embryos (Hhat ${ }^{\text {creface }}$ Ptch1 wiggable embryos) exhibit partially rescued craniofacial patterning presumably as a result of restoring SHH signaling to normal levels. However, Hhat refface Ptch 1wiggable embryos still displayed cleft lip with a primary palate bone cleft (Figure 1, B and E). There are several studies that have described secondary cleft palate via diminished 

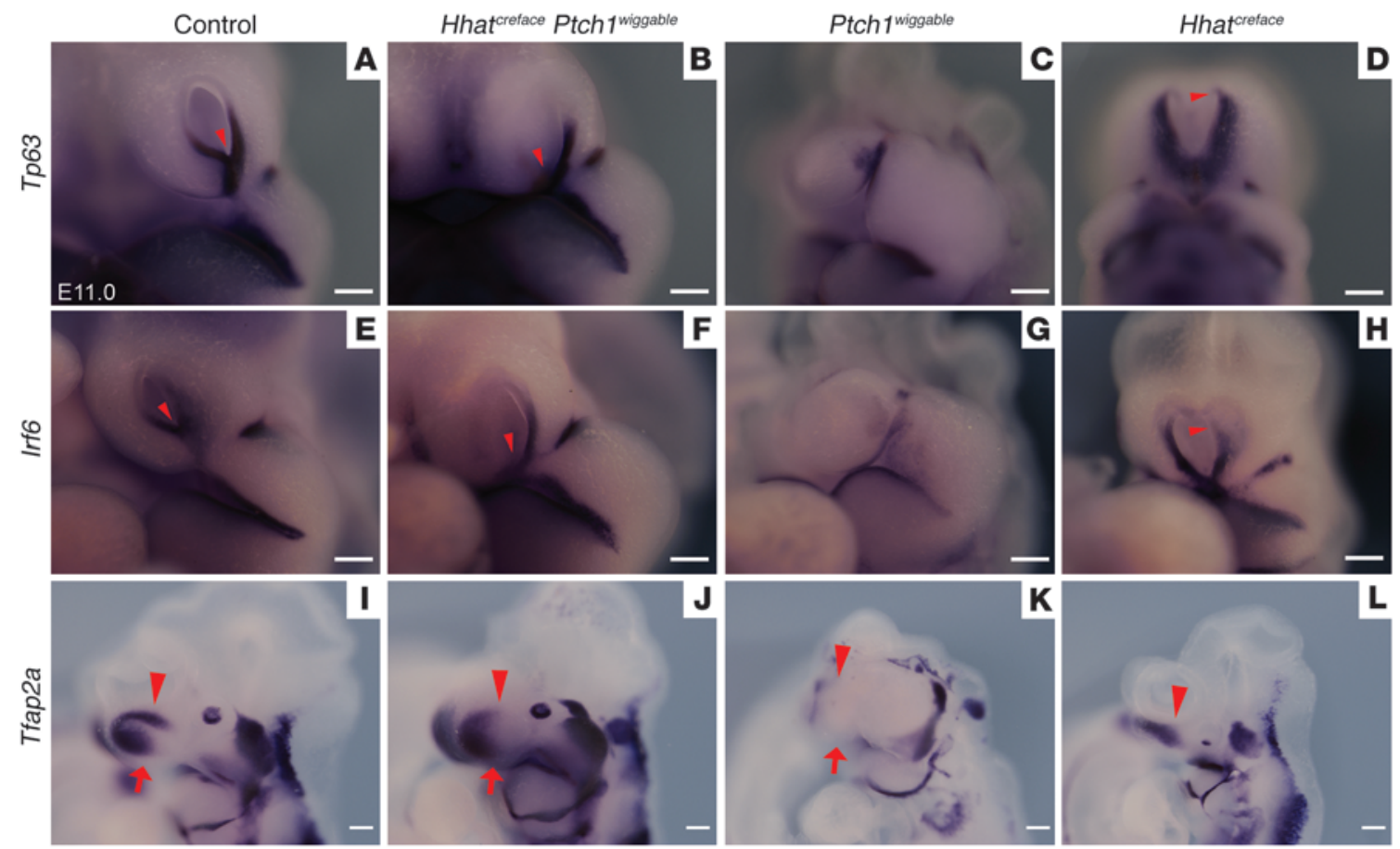

\section{Figure 7}

p63/IRF6 signaling pathway and Tfap2a expression in the FNP are affected by altering SHH signaling. (A-D) Tp63 expression in E11.0 embryos. (A) Tp63 is strongly expressed at the lambdoidal region in control embryos (red arrowhead; orientation is the same as that shown in Figure 2, I-L). (B) Hhatcreface Ptch1wiggable embryos showed reduced Tp63 expression at the lambdoidal region (red arrowhead), and (C) Ptch1wiggable embryos showed an even lower expression level. (D) Conversely, Hhatcreface embryos showed expanded Tp63 expression in LNP (red arrowhead). (E-H) Irf6 expression in E11.0 embryos. (E) Irf6 expression at the lambdoidal region in control embryos is similar to Tp63 expression (red arrowhead). Irf6 expression was downregulated in both (F) Hhatcreface Ptch1wiggable (red arrowhead) and (G) Ptch1wiggable embryos. (H) Similar to Tp63, Irf6 showed expanded expression in Hhatcreface embryos (red arrowhead; orientation is the same as that shown in Figure 2, I-L). (I-L) Tfap2a expression in E11.0 embryos. (I) Tfap2a was expressed at mesenchymal cells in MNP (red arrow in I-L) and LNP (red arrowheads in I-L) in control embryos. The expression was attenuated in (K) Ptch1wiggable and (J) Hhatcreface Ptch1 wiggable embryos, (L) while Hhatcreface embryos maintained expression (orientation is the same as that shown in Figure 2, E-H). Scale bars: $200 \mu \mathrm{m}$.

or elevated SHH signaling $(9,10,33,34)$. However, the mechanisms underpinning the pathogenesis of cleft lip arising from altered SHH signaling are still poorly understood. Detailed observations of the developing face of Hhat ${ }^{\text {treface }}$ Ptch1 $1^{\text {wiggable }}$ embryos revealed hypoplastic nasal process development at E11.0, leading to pathogenesis of a cleft between the MNPs and LNPs at E11.5, which suggests that growth as well as fusion of these processes was impaired (Figure 2, F, J, and N). It is well known that during nasal process fusion epithelial cells need to be removed in order to form a mesenchymal bridge, which is critical for a rigid connection in the developing lip and primary palate (3). From histological sections of the lambdoidal region, we discovered that Hhatcreface

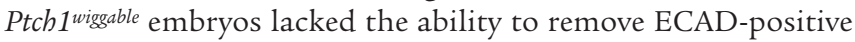
epithelial cells from the tip of developing processes (Figure 3, F and L). Furthermore, Hhat ${ }^{\text {reface }}$ Ptch $1^{\text {wiggable }}$ embryos showed reduced epithelial cell death (Figure 3F, white arrow) and increased epithelial proliferation in association with the persistence of ECAD-labeled epithelia (Figure 3, L and O). Although the ratio of cell death to total epithelial seam cells did not show a statistically significant difference between control and Hhat ${ }^{\text {reface }}$ Ptch1 ${ }^{\text {wiggable }}$ embryos (Figure 3P), we postulate that the reduction of cell death in Hhat ${ }^{\text {creface }}$ Ptch1wiggable embryos affects the balance between cell proliferation and cell death, resulting in a persistent epithelial seam. Our work is consistent with other studies showing the persistence of epithelial cells as a consequence of decreased cell death or increased proliferation in association with CL/P $(13-15,35,36)$.

Precise levels and distribution of $\mathrm{SHH}$ signaling are necessary for craniofacial development. In order to assess how SHH signaling was affected in each mutant, we performed in situ hybridization for Ptch1 and Gli1, since they are direct targets of the SHH signaling cascade. During normal craniofacial development, Ptch1 and Gli1 were highly expressed in midline facial fissures and formed a gradient toward the lateral side of the face with no expression in the LNPs (Figure 4, I and M). Expression of both Ptch1 and Gli1 was noticeably upregulated in the FNPs and expanded toward the LNPs in Ptch1 1wiggable embryos (Figure 4, K and O). The Ptch1 wiggable mutation causes a C-terminal truncation of $\mathrm{PTCH} 1$ protein, which is one of the key receptors of SHH. It is well known that the C-terminal intracellular domain of PTCH1 is responsible for suppressing smoothened activity by forming heterodimers in the absence of $\mathrm{HH}$ ligands (37). These results clearly explain the reason that SHH signaling is overactivated in Ptch1 wigable embryos in the absence of SHH ligand.

In contrast, Hhat creface embryos showed noticeably reduced $\mathrm{SHH}$ signaling during facial development (Figure 4, $\mathrm{L}$ and P). This is because Hhat treface embryos are unable to palmitoylate $\mathrm{SHH}$, which 

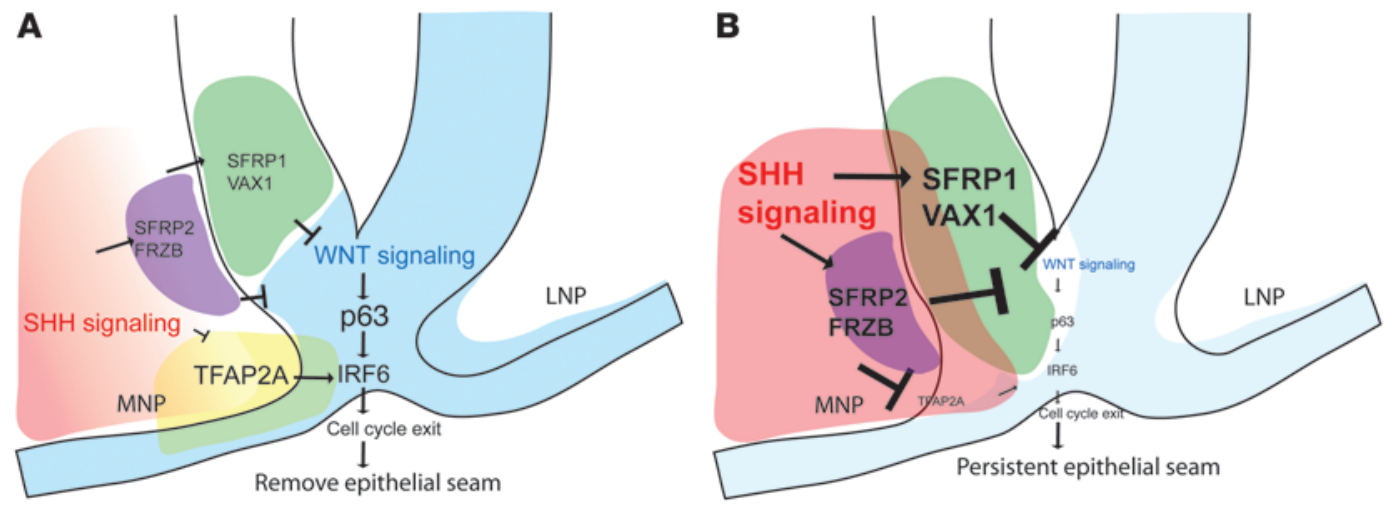

\section{Figure 8}

Schematic summary of the critical role of SHH signaling in nasal processes growth and fusion. (A) SHH signaling is important to the removal of the epithelial seam between the nasal processes by maintaining the p63/IRF6 signaling cascade via proper WNT signaling and Tfap2a expression. (B) Expanded SHH signaling leads to cleft lip phenotype by disrupting various signaling cascades required for epithelial seam breakdown.

diminishes its capacity for diffusion and limits the SHH signaling gradient activity $(18,20,21)$. Our results demonstrate that facial development can be partially restored in double-homozygous embryos (Figure 2). The total amount of Ptch1 and Gli1 expression in the maxillary complex of Hhatcreface Ptch1 wiggable embryos was restored (Supplemental Figure 3, B and C); however, their domain of activity revealed persistent expression in the LNPs, suggesting that the distribution of SHH signaling was still affected (Figure 4 , $\mathrm{J}$ and $\mathrm{N}$ ). These results clearly demonstrate that craniofacial morphology is altered in association with the strength and pattern of Shb signaling.

Interaction of SHH and canonical WNT signaling during craniofacial development. Similar to SHH signaling, canonical WNT signaling is also well known to be a critical regulator of craniofacial development (38). Previous reports have shown that reduced canonical WNT signaling through deletion of Wnt $9 b$ or Lrp6 during craniofacial development leads to facial deformity, including CL/P, in mice $(13,14)$. Furthermore, a strong correlation between ectodermal canonical WNT signaling and nasal process growth has been reported (39). From our observations of the patterns of SHH signaling (Ptch1-LacZ) and canonical WNT signaling (TOPgal) during craniofacial development, we found that those 2 signaling pathways complement each other during facial development (Supplemental Figure 4). Furthermore, our RNA-seq data revealed that some canonical WNT signaling genes, such as Axin2 and Tcf4, are inversely expressed relative to Gli1 and Ptch1, which are targets of SHH signaling (Supplemental Figure 3). These findings led us to hypothesize that $\mathrm{SHH}$ signaling represses canonical WNT signaling in order to generate defined facial compartments during craniofacial development. By crossing all mutants to TOPgal mice and assessing $L a c Z$ expression, we observed enhanced canonical WNT signaling in the growing LNPs of Hhat treface embryos, which exhibited reduced SHH signaling from E10.0 to E10.5 (Figure 5, $\mathrm{D}$ and $\mathrm{H}$ ). Conversely, attenuated canonical WNT signaling was observed in Ptch1 $1^{\text {wiggable }}$ and Hhat ${ }^{\text {creface }}$ Ptch 1 $^{\text {wiggable }}$ embryos in concert with expanded SHH signaling (Figure 5, F and G). Importantly, at E11.0, when the MNP and LNP begin to fuse, the lambdoidal region expressed high levels of Wnt activity (Figure 5I). However, this activity was considerably reduced in Hhat creface Ptch1 wiggable embryos (Figure 5J). This reduction of canonical WNT signaling in the lambdoidal region is also observed in other mice in association with cleft lip $(13,14,40)$. Consistent with these observations, we could also reproduce this phenotype by culturing whole embryos in the presence of a SHH signaling agonist (Supplemental Figure 5). These results clearly show that SHH signaling inhibits canonical WNT signaling during the process of normal lip fusion.

Canonical WNT inhibitory gene activity is altered by modulating $\mathrm{SHH}$ signaling. Since there was a clear inhibition of canonical WNT activity by increased SHH activity during craniofacial development, we explored a role for canonical WNT inhibitory genes in this process. Numerous canonical WNT inhibitory genes have been reported (41), and 2 classes of secreted factor exist. One is the dickkopf (Dkk) class and another is the secreted frizzled-related (Sfrp) class (42). We interrogated our RNA-seq data in combination with the literature to identify factors, which were activated by enhanced SHH signaling. As a result, we focused on Vax1 and Sfrp family genes. Recent studies reported significant genetic associations between VAX1 mutations and CL/P in humans (43-45). Furthermore, Vax1-null mice display cleft palate (46). Vax2 has been shown to be activated by SHH signaling (47), and Vax1, together with Vax2, regulate a dominant-negative truncated transcription factor, $T c f 7 l 2$, to antagonize canonical WNT signaling (48). In the present study, we observed altered Vax1 expression in Hhatcreface Ptch $1^{\text {wiggable }}$ embryos, particularly in the MNP close to the region in which process fusion takes place (Figure 6B).

Sfrp1 and Sfrp2 double-null mice display craniofacial deformity, illustrating their important roles during craniofacial development $(49,50)$. Sfrp1 transcription has been shown to be activated by SHH signaling during retinal development (51), and Sfrp2 is activated by SHH and plays a role in restricting canonical WNT signaling during neural tube pattering (52). In the present study, we found altered Sfrp1 expression in the vicinity of the lambdoidal region in Hhat ${ }^{\text {creface }}$ Ptch $1^{\text {wiggable }}$ embryos similar to that of Vax1 (Figure 6F). Sfrp2 and Frzb showed increased activity in the MNPs of Hhatcreface Ptch1 ${ }^{\text {wiggable }}$ embryos (Figure 6, J and N). These results clearly demonstrate that diminished canonical WNT signaling in the lambdoidal junction in Hhat creface Ptch $1^{\text {wiggable }}$ embryos correlates with altered or increased expression of canonical WNT inhibitory genes mediated by SHH signaling. We identified GLI2 and GLI3 consensus binding sequences in potential regulatory regions of 
Sfrp2 and Sfrp1, respectively. However, these sequences are not conserved among species, and it remains unknown whether these WNT inhibitory genes are direct targets of SHH signaling. Nonetheless, disrupting WNT signaling is tightly associated with the etiology of CL/P both in mice and humans (12-14, 40). Taken together, our results highlight the indispensable role of SHH signaling in controlling canonical WNT signaling during nasal process growth and fusion via multiple WNT inhibitory genes. It is also important to note, however, the possibility that modification of SHH signaling could affect the growth of each MNP and LNP, resulting in the nasal epithelium coming into closer proximity with the highly hypoplastic bridge at the seam.

A novel pathway in the pathogenesis of cleft lip. Tp63 disruption leads to $\mathrm{CL} / \mathrm{P}$ in mice (24) and is also known to be responsible for certain human syndromes that display CL/P as part of the phenotype $(25,53)$. Irf6 has also been identified as a candidate gene for isolated CL/P as well as Van der Woude's syndrome (54). Interestingly, recent studies demonstrated that p63 directly activates Irf6 transcription, allowing epithelial cells to exit the cell cycle during proper processes fusion $(16,17,36,55)$. Furthermore, inhibiting the p63/IRF6 signaling cascade had been shown to facilitate the persistence of excessive periderm cells in the secondary palate, which is mechanistically associated with the pathogenesis of CL/P (17). In the present study, we confirmed Irf6 downregulation in the lambdoidal region, together with attenuated Tp63 expression, in Hhatcreface Ptch1 wiggable and Ptch1 ${ }^{\text {wiggable }}$ embryos (Figure 7, B, C, F, and G, and Supplemental Figure 6, B, C, F, and G). Taken together with increased cell proliferation (Figure 3, K, L, and $\mathrm{O}$ ) and stronger SSEA1 expression (Supplemental Figure 2) in the epithelium of the nasal process in Hhat ${ }^{\text {creface }}$ Ptch $1^{\text {wiggable }}$ embryos, our data strongly suggest that the failure of p63/IRF6-dependent cell cycle exit and periderm cell maintenance is part of the etiology and pathogenesis of cleft lip. Furthermore, a recent publication revealed a possible role for canonical WNT signaling in activating the p63/IRF6 signaling cascade (15), which is consistent with our finding of attenuated WNT signaling in association with upregulated or expanded SHH signaling (Figure 5, J and K). We also investigated the influence of altering SHH signaling on neural crest cell (NCC) behavior, since many craniofacial defects, including CL/P, are known to be tightly associated with defects in NCC development (56). Crabp1 and Snail1 are broadly expressed by CNCs $(57,58)$; however, we did not observe any significant differences in Crabp1 or Snail1 expression in the FNPs of mutant embryos compared with control embryos (Supplemental Figure 7). Interestingly, another NCC marker, Tfap $2 a$, which is a transcription factor well known for its critical roles during craniofacial development $(59,60)$, was considerably reduced in the FNPs of Ptch $1^{\text {wiggable }}$ embryos and was not restored to normal levels in Hhatcreface Ptch1 1 wiggable embryos (Figure 7, J and K, and Supplemental Figure 6, J and K). These results strongly suggest that proper $\mathrm{SHH}$ signaling is indispensable for NCC patterning especially via Tfap $2 a$ during normal nasal process growth and fusion. We also confirmed reduced TFAP2A activity in epithelial seam cells in Hhat ${ }^{\text {reface }}$ Ptch1 $1^{\text {wiggable }}$ and Ptch1 $1^{\text {wiggable }}$ embryos compared with that in control embryos (Supplemental Figure 6, M, N, and O). Interestingly, in humans, disruption of TFAP2A leads to branchio-oculo-facial syndrome, which is a congenital disease associated with CL/P (61). Moreover, disruption of the TFAP2A binding site in the promoter of IRF6 is associated with the etiology of cleft lip in humans (30).
Collectively, our results demonstrate a novel mechanism whereby SHH signaling modulates WNT signaling and TFAP2A expression via a p63/IRF6 cascade during facial process growth, morphogenesis, and fusion (Figure 8). Disruption of any component can result in cleft lip/palate, highlighting the signaling complexity that is required for normal nasal process growth and fusion.

\section{Methods}

Mouse lines. Ptch1 $1^{\text {wiggable }}$ mice were generated through $\mathrm{N}$-ethyl- $\mathrm{N}$-nitrosourea mutagenesis in our lab (19). Hhatereface, TOPgal, and Ptch1-LacZ mice were maintained as described previously $(18,22,23)$. For embryonic staging, the morning of vaginal plug identification was defined as E0.5. We designated embryos carrying homozygous mutations as Hhatcreface or Ptch1 $1^{\text {wiggable }}$, respectively, and Hhat ${ }^{\text {creface }}$ Ptch1 ${ }^{\text {wiggable }}$ as double-homozygous mice. Control mice described in the present study were either wild-type or heterozygous littermates.

Skeletal preparations. Embryos were collected at E16.5. Cartilage and bone were visualized by staining with Alizarin red and Alcian blue as described previously (18).

Whole-mount nuclear fluorescent imaging. For analyzing facial morphological structure, we fixed the embryos in $4 \%$ PFA overnight at $4{ }^{\circ} \mathrm{C}$. Fixed embryos were washed several times in PBS and stained directly with DAPI (Sigma-Aldrich) $(1: 1,000)$ in PBS overnight at $4{ }^{\circ} \mathrm{C}$ and visualized and photographed as previously described (62).

Immunohistochemistry and cell death detection. Frozen sections (10- $\mu \mathrm{m}$ thick) were mounted on adhesive slides. The slides were washed in TBST (TBS with $0.1 \%$ Tween) and blocked with $1 \%$ BSA in TBST for 1 hour at room temperature. Slides were incubated with anti-ECAD (Abcam), anti-phospho-Histone-3 (Upstate/Millipore), anti-TFAP2A (3B5, Developmental Studies Hybridoma Bank), and anti-SSEA1 (Santa Cruz Biotechnology). Antibodies were diluted 1:500 for ECAD and phospho-Histone-3, 1:50 for TFAP2A, and 1:200 for SSEA1 overnight at $4{ }^{\circ} \mathrm{C}$ and washed several times in TBST. The slides were incubated using the appropriate secondary antibody for 1 hour at room temperature and counterstained with DAPI (Sigma-Aldrich) (1:1,000) for 10 minutes at room temperature and mounted with fluorescent mounting medium (DakoCytomation). To visualize apoptosis in conjunction with ECAD immunostaining, we used the In Situ Cell Death Detection Kit Fluorescein (Roche) according to the manufacturer's instructions.

In situ hybridization. Embryos were collected as described above and fixed overnight in $4 \%$ PFA at $4{ }^{\circ} \mathrm{C}$. For whole-mount in situ hybridization (WISH), the samples were dehydrated in graded methanol and stored at $-20^{\circ} \mathrm{C}$ until use. We rehydrated the samples and performed standard WISH protocol, which has been described before (63). Some whole-mount embryos labelled by in situ hybridization were processed were processed through a sucrose gradient, embedded in Tissue-Tek (OCT compound, Sakura), and cut into $18-\mu \mathrm{m}$-thick sections for detailed observation. Section in situ hybridization was performed using $10-\mu \mathrm{m}$-thick frozen sections. Hybridization and color development were performed as previously reported (64). The constructs used to produce Tp63 and Irf6 as well as Vax1 riboprobes were provided by Licia Selleri (Cornell University Medical School, New York, New York, USA) and Douglas J. Epstein (University of Pennsylvania, Philadelphia, Pennsylvania, USA), respectively.

RNA-seq. Total RNA was isolated from the E11.5 maxillary complex (encompassing MNP, LNP, and maxillary process) of embryos of each genotype (Supplemental Figure 3A) using the RNeasy Kit (Qiagen) according to the manufacturer's protocol. Sequences for each RNA sample were aligned using TopHat (v1.4.0). The reference genome and annotation files used for the alignment were from the UCSC $\mathrm{mm} 9$ genome and the ensemble build 65 . Gene expression levels for genes are calculated using cufflinks 
(v1.3.0) $(65,66)$ with TopHat-aligned reads. We generated read counts that were mapped to exons of genes using BEDTOOLs.

$\beta$-Galactosidase staining. Hhat ${ }^{\text {treface }}$ Ptch1 wiggable $^{\text {mice were mated to TOPgal }}$ mice (23), and embryos were collected at E10.0, E10.5, and E11.0. All embryos were stained using the $\beta$-Galactosidase Staining Solution Kit (Chemicon/ Millipore) according to the manufacturer's instructions. Some of stained embryos were processed for 18 - $\mu \mathrm{m}$-thick frozen sections for detailed observation as described above.

Whole embryo culture with Shh agonist. TOPgal embryos were obtained from pregnant female mice at E10.0 and placed into Tyrode's solution. The decidua and Reichert's membrane were carefully removed, and a minimal incision was made in yolk sac and amnion to expose the head of the embryos. The embryos were transferred into $37^{\circ} \mathrm{C} 65 \%$ oxygen gas-supplied whole embryo culture rat serum (Harlan) with $2 \mathrm{mg} / \mathrm{ml} \mathrm{D-(+)-Glucose} \mathrm{solution} \mathrm{(Sigma-Aldrich)}$ (67). $10 \mu \mathrm{M}$ Purmorphamine (Millipore) was added to the culture media as an agonist of SHH signaling. After 24-hour culture, the embryos were collected and processed though $\beta$-galactosidase staining as described above.

Statistics. Two-tailed Student's $t$ tests were performed for data represented in Figure 3O. $P$ values in Supplemental Figure 3 were adjusted using the Benjamini-Hochberg procedure to control the false discovery rate. An R package, DESeq, was used to find differentially expressed genes between control and mutant samples. $P$ values of less than 0.05 were considered significant in all experiments.

Study approval. Mice were housed in the Laboratory Animal Services Facility at the Stowers Institute for Medical Research. Welfare guidelines and procedures were performed with approval of the Stowers Institute for Medical Research IACUC.

1. van der Meulen JC. Oblique facial clefts: pathology, etiology, and reconstruction. Plast Reconstr Surg. 1985;76(2):212-224

2. Ray HJ, Niswander L. Mechanisms of tissue fusion during development. Development. 2012. 139(10):1701-1711.

3. Jiang R, Bush JO, Lidral AC. Development of the upper lip: morphogenetic and molecular mechanisms. Dev Dyn. 2006;235(5):1152-1166.

4. Nawshad A, Hay ED. TGFbeta3 signaling activates transcription of the LEF1 gene to induce epithelial mesenchymal transformation during mouse palate development. J Cell Biol. 2003;163(6):1291-1301.

5. Iseki S. Disintegration of the medial epithelial seam: Is cell death important in palatogenesis? Dev Growth Differ. 2011;53(2):259-268.

6. Dixon MJ, Marazita ML, Beaty TH, Murray JC. Cleft lip and palate: understanding genetic and environmental influences. Nat Rev Genet. 2011; 12(3):167-178.

7. Richieri-Costa A, Ribeiro LA. Holoprosencephaly and holoprosencephaly-like phenotypes: Review of facial and molecular findings in patients from a craniofacial hospital in Brazil. Am J Med Genet C Semin Med Genet. 2010;154C(1):149-157.

8. Mansilla MA, et al. Contributions of PTCH gene variants to isolated cleft lip and palate. Cleft Palate Craniofac J. 2006;43(1):21-29.

9. Rice R, et al. Disruption of Fgf10/Fgfr2b-coordinated epithelial-mesenchymal interactions causes cleft palate. J Clin Invest. 2004;113(12):1692-1700.

10. Lan Y, Jiang R. Sonic hedgehog signaling regulates reciprocal epithelial-mesenchymal interactions controlling palatal outgrowth. Development. 2009;136(8):1387-1396.

11. Lipinski RJ, et al. Cleft lip and palate results from Hedgehog signaling antagonism in the mouse: Phenotypic characterization and clinical implications. Birth Defects Res A Clin Mol Teratol. 2010; 88(4):232-240.

12. Chiquet BT, et al. Variation in WNT genes is associated with non-syndromic cleft lip with or without

\section{Acknowledgments}

The authors thank members of the Trainor lab for their insights and constructive comments throughout the course of this project and completion of this paper. We are indebted to Melissa Childers for excellent care and maintenance of our mouse colony; Nancy Thomas, Nannette Marsh, and Karen Smith for histological sections; and Karin Zueckert-Gaudenz and Hua Li for transcriptome analysis. We are also very grateful for the generosity of Danielle Manning, who generated the AP2-Cre (Creface) mouse line, and Jennifer Dennis, who identified and characterized the location of

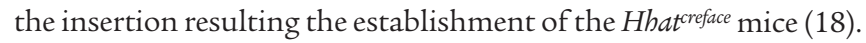
We also deeply appreciate Licia Selleri and Douglas J. Epstein for providing constructs for in situ hybridizations. Research in the Trainor laboratory is supported by the Stowers Institute for Medical Research and National Institute of Dental and Craniofacial Research (DE016082). The Williams laboratory is funded by the National Institute of Dental and Craniofacial Research (DE019843 and DE012728). The Iulianella laboratory is funded by the Natural Sciences and Engineering Council of Canada (grant 386595).

Received for publication August 13, 2013, and accepted in revised form January 2, 2014.

Address correspondence to: Paul A. Trainor, Stowers Institute for Medical Research, 1000 East 50th Street, Kansas City, Missouri 64110, USA. Phone: 816.926.4414; Fax: 816.926.2051; E-mail: PAT@stowers.org.

cleft palate. Hum Mol Genet. 2008;17(14):2212-2218.

13. Song L, et al. Lrp6-mediated canonical Wnt signaling is required for lip formation and fusion. Development. 2009;136(18):3161-3171.

14. Jin Y-R, Han XH, Taketo MM, Yoon JK. Wnt9b-dependent FGF signaling is crucial for outgrowth of the nasal and maxillary processes during upper jaw and lip development. Development. 2012; 139(10):1821-1830.

15. Ferretti E, et al. A conserved Pbx-Wnt-p63-Irf6 regulatory module controls face morphogenesis by promoting epithelial apoptosis. Dev Cell. 2011; 21(4):627-641.

16. Moretti F, et al. A regulatory feedback loop involving p 63 and IRF 6 links the pathogenesis of 2 genetically different human ectodermal dysplasias. JClin Invest. 2010;120(5):1570-1577.

17. Thomason HA, et al. Cooperation between the transcription factors p63 and IRF6 is essential to prevent cleft palate in mice. J Clin Invest. 2010; 120(5):1561-1569.

18. Dennis JF, et al. Mutations in Hedgehog acyltransferase (Hhat) perturb Hedgehog signaling, resulting in severe acrania-holoprosencephaly-agnathia craniofacial defects. PLoS Genet. 2012;8(10):e1002927.

19. Sandell LL, et al. A phenotype-driven ENU mutagenesis screen identifies novel alleles with functional roles in early mouse craniofacial development. Genesis. 2011;49(4):342-359.

20. Ohlig S, et al. Sonic hedgehog shedding results in functional activation of the solubilized protein. Dev Cell. 2011;20(6):764-774.

21. Chen M-H, Li Y-J, Kawakami T, Xu S-M, Chuang $\mathrm{P}-\mathrm{T}$. Palmitoylation is required for the production of a soluble multimeric Hedgehog protein complex and long-range signaling in vertebrates. Genes Dev. 2004;18(6):641-659.

22. Goodrich LV, Milenkovic L, Higgins KM, Scott MP. Altered neural cell fates and medulloblastoma in mouse patched mutants. Science. 1997; 277(5329):1109-1113.

23. DasGupta R, Fuchs E. Multiple roles for activated
LEF/TCF transcription complexes during hair follicle development and differentiation. Development. 1999;126(20):4557-4568.

24. Thomason HA, Dixon MJ, Dixon J. Facial clefting in Tp63 deficient mice results from altered Bmp4, Fgf8 and Shh signaling. Dev Biol. 2008;321(1):273-282.

25 . Ferone $G$, et al. Mutant p 63 causes defective expansion of ectodermal progenitor cells and impaired FGF signalling in AEC syndrome. EMBO Mol Med. 2012;4(3):192-205.

26. Achilleos A, Trainor PA. Neural crest stem cells: discovery, properties and potential for therapy. Cell Res. 2012;22(2):288-304.

27. Santagati F, Rijli FM. Cranial neural crest and the building of the vertebrate head. Nat Rev Neurosci. 2003;4(10):806-818.

28. Bhatt S, Diaz R, Trainor PA. Signals and switches in Mammalian neural crest cell differentiation. Cold Spring Harb Perspect Biol. 2013;5(2):a008326.

29. Jeong J, Mao J, Tenzen T, Kottmann AH, McMahon AP. Hedgehog signaling in the neural crest cells regulates the patterning and growth of facial primordia. Genes Dev. 2004;18(8):937-951.

30. Rahimov F, et al. Disruption of an AP- $2 \alpha$ binding site in an IRF6 enhancer is associated with cleft lip. Nat Genet. 2008;40(11):1341-1347.

31. Ming JE, Roessler E, Muenke M. Human developmental disorders and the Sonic hedgehog pathway. Mol Med Today. 1998;4(8):343-349.

32. Sekhon PS, Ethunandan M, Markus AF, Krishnan $G$, Rao CB. Congenital anomalies associated with cleft lip and palate-an analysis of 1623 consecutive patients. Cleft Palate Craniofac J. 2011;48(4):371-378.

33. Huang X, Goudy SL, Ketova T, Litingtung Y, Chiang C. Gli3-deficient mice exhibit cleft palate associated with abnormal tongue development. Dev Dyn. 2008;237(10):3079-3087.

34. Cobourne MT, et al. Sonic hedgehog signalling inhibits palatogenesis and arrests tooth development in a mouse model of the nevoid basal cell carcinoma syndrome. Dev Biol. 2009;331(1):38-49.

35. Xu X, et al. Cell autonomous requirement for Tgfbr2 
in the disappearance of medial edge epithelium during palatal fusion. Dev Biol. 2006;297(1):238-248.

36. Iwata J, et al. Smad4-Irf6 genetic interaction and TGFbeta-mediated IRF6 signaling cascade are crucial for palatal fusion in mice. Development. 2013; 140(6):1220-1230.

37. Stone DM, et al. The tumour-suppressor gene patched encodes a candidate receptor for Sonic hedgehog. Nature. 1996;384(6605):129-134

38. Brugmann SA, et al. Wnt signaling mediates regional specification in the vertebrate face. Development. 2007;134(18):3283-3295.

39. Reid BS, Yang H, Melvin VS, Taketo MM, Williams $\mathrm{T}$. Ectodermal Wnt $/ \beta$-catenin signaling shapes the mouse face. Dev Biol. 2011;349(2):261-269.

40. Yamada W, et al. Craniofacial malformation in R-spondin 2 knockout mice. Biochem Biophys Res Commun. 2009;381(3):453-458.

41. Cruciat CM, Niehrs C. Secreted and transmembrane wnt inhibitors and activators. Cold Spring Harb Perspect Biol. 2013;5(3):a015081.

42. Kawano Y, Kypta R. Secreted antagonists of the Wnt signalling pathway. J Cell Sci. 2003; 116(pt 13):2627-2634.

43. Slavotinek AM, et al. VAX1 mutation associated with microphthalmia, corpus callosum agenesis, and orofacial clefting: The first description of a VAX1 phenotype in humans. Hum Mutat. 2012;33(2):364-368.

44. Butali A, et al. Replication of genome wide association identified candidate genes confirm the role of common and rare variants in PAX7 and VAX1 in the etiology of nonsyndromic CL(P). Am J Med Genet A. 2013;161(5):965-972.

45. de Aquino S, et al. Polymorphisms in FGF12, VCL, CX43, and VAX1 in Brazilian patients with nonsyndromic cleft lip with or without cleft palate. BMC Med Genet. 2013;14:53.

46. Bertuzzi S, Hindges R, Mui SH, O’Leary DDM, Lemke G. The homeodomain protein Vax1 is required for axon guidance and major tract forma- tion in the developing forebrain. Genes Dev. 1999; 13(23):3092-3105

47. Kim JW, Lemke G. Hedgehog-regulated localization of Vax 2 controls eye development. Genes Dev. 2006;20(20):2833-2847.

48. Vacik T, Stubbs JL, Lemke G. A novel mechanism for the transcriptional regulation of Wnt signaling in development. Genes Dev. 2011;25(17):1783-1795.

49. Satoh W, Gotoh T, Tsunematsu Y, Aizawa S, Shimono A. Sfrp1 and Sfrp2 regulate anteroposterior axis elongation and somite segmentation during mouse embryogenesis. Development. 2006;133(6):989-999.

50. Satoh W, Matsuyama M, Takemura H, Aizawa S, Shimono A. Sfrp1, Sfrp2, and Sfrp5 regulate the Wnt/ $\beta$-catenin and the planar cell polarity pathways during early trunk formation in mouse. Genesis. 2008;46(2):92-103.

51. Borday C, et al. Antagonistic cross-regulation between Wnt and Hedgehog signalling pathways controls post-embryonic retinal proliferation. Development. 2012;139(19):3499-3509.

52. Lee CS, Buttitta LA, May NR, Kispert A, Fan CM. SHH-N upregulates Sfrp2 to mediate its competitive interaction with WNT1 and WNT4 in the somitic mesoderm. Development. 2000;127(1):109-118.

53. Celli J, et al. Heterozygous germline mutations in the p53 homolog p63 are the cause of EEC syndrome. Cell. 1999;99(2):143-153.

54. Zucchero TM, et al. Interferon regulatory factor 6 (IRF6) gene variants and the risk of isolated cleft lip or palate. N Engl J Med. 2004;351(8):769-780.

55. Fakhouri WD, et al. MCS9.7 enhancer activity is highly, but not completely, associated with expression of Irf6 and p63. Dev Dyn. 2012;241(2):340-349.

56. Trainor PA. Craniofacial birth defects: The role of neural crest cells in the etiology and pathogenesis of Treacher Collins syndrome and the potential for prevention. AmJ Med Genet A. 2010;152A(12):2984-2994.

57. Dencker L, Annerwall E, Busch C, Eriksson U. Localization of specific retinoid-binding sites and expression of cellular retinoic-acid-binding protein (CRABP) in the early mouse embryo. Development. 1990;110(2):343-352.

58. Murray SA, Oram KF, Gridley T. Multiple functions of Snail family genes during palate development in mice. Development. 2007;134(9):1789-1797.

59. Mitchell PJ, Timmons PM, Hébert JM, Rigby PWJ, Tjian R. Transcription factor AP-2 is expressed in neural crest cell lineages during mouse embryogenesis. Genes Dev. 1991;5(1):105-119.

60. Zhang J, et al. Neural tube, skeletal and body wall defects in mice lacking transcription factor AP-2. Nature. 1996;381(6579):238-241.

61. Milunsky JM, et al. TFAP2A mutations result in branchio-oculo-facial syndrome. Am J Hum Genet. 2008;82(5):1171-1177.

62. Sandell LL, Kurosaka H, Trainor PA. Whole mount nuclear fluorescent imaging: convenient documentation of embryo morphology. genesis. 2012; 50(11):844-850

63. Nagy A, Gertsenstein M, Vintersten K, Behringer RR. Manipulating The Mouse Embryo: A Laboratory Manual. 3rd ed. Cold Spring Harbor, New York, USA; Cold Spring Harbor Laboratory; 2003.

64. Iseki S, Wilkie AO, Heath JK, Ishimaru T, Eto K, Morriss-Kay GM. Fgfr2 and osteopontin domains in the developing skull vault are mutually exclusive and can be altered by locally applied FGF2. Development. 1997;124(17):3375-3384.

65. Trapnell C, Pachter L, Salzberg SL. TopHat: discovering splice junctions with RNA-Seq. Bioinformatics. 2009;25(9):1105-1111.

66. Trapnell C, et al. Transcript assembly and quantification by RNA-Seq reveals unannotated transcripts and isoform switching during cell differentiation. Nat Biotechnol. 2010;28(5):511-515.

67. Trainor PA, Sobieszczuk D, Wilkinson D, Krumlauf R. Signalling between the hindbrain and paraxial tissues dictates neural crest migration pathways. Development. 2002;129(2):433-442. 